\title{
Bacterial Effector HopF2 Suppresses Arabidopsis Innate Immunity at the Plasma Membrane
}

\author{
Shujing Wu, ${ }^{1}$ Dongping Lu, ${ }^{2}$ Mehdi Kabbage, ${ }^{1}$ Hai-Lei Wei, ${ }^{3}$ Bryan Swingle,,${ }^{4,3}$ Angela R. Records, ${ }^{1}$ \\ Martin Dickman, ${ }^{1}$ Ping He, ${ }^{2}$ and Libo Shan ${ }^{1}$ \\ ${ }^{1}$ Department of Plant Pathology and Microbiology and ${ }^{2}$ Department of Biochemistry and Biophysics, and Institute for Plant \\ Genomics and Biotechnology, Texas A\&M University, College Station, TX 77843, U.S.A.; ${ }^{3}$ Department of Plant Pathology \\ and Plant-Microbe Biology, Cornell University, and 4 United States Department of Agriculture-Agricultural Research Service, \\ Ithaca, NY 14853, U.S.A.
}

Submitted 2 July 2010. Accepted 27 December 2010.

\begin{abstract}
Many bacterial pathogens inject a cocktail of effector proteins into host cells through type III secretion systems. These effectors act in concert to modulate host physiology and immune signaling, thereby promoting pathogenicity. In a search for additional Pseudomonas syringae effectors in suppressing plant innate immunity triggered by pathogen or microbe-associated molecular patterns (PAMPs or MAMPs), we identified $P$. syringae tomato DC3000 effector HopF2 as a potent suppressor of early immune-response gene transcription and mitogen-activated protein kinase (MAPK) signaling activated by multiple MAMPs, including bacterial flagellin, elongation factor Tu, peptidoglycan , lipopolysaccharide and HrpZ1 harpin, and fungal chitin. The conserved surfaceexposed residues of HopF2 are essential for its MAMP suppression activity. HopF2 is targeted to the plant plasma membrane through a putative myristoylation site, and the membrane association appears to be required for its MAMP-suppression function. Expression of HopF2 in plants potently diminished the flagellin-induced phosphorylation of BIK1, a plasma membrane-associated cytoplasmic kinase that is rapidly phosphorylated within one minute upon flagellin perception. Thus, HopF2 likely intercepts MAMP signaling at the plasma membrane immediately of signal perception. Consistent with the potent suppression function of multiple MAMP signaling, expression of $\mathrm{HopF2}$ in transgenic plants compromised plant nonhost immunity to bacteria $P$. syringae pv. Phaseolicola and plant immunity to the necrotrophic fungal pathogen Botrytis cinerea.
\end{abstract}

Plants have evolved robust immune systems to defend against pathogen threats. The innate immune responses are launched by recognition of conserved pathogen or microbeassociated molecular patterns (PAMPs or MAMPs) through host pattern-recognition receptors (Boller and Felix 2009; Jones and Dangl 2006). In plants, the plasma membrane localized leucine-rich repeat receptor-like kinases (LRR-RLK) play a pivotal role in detecting MAMPs and mount PAMP-triggered immunity (PTI). Two well-characterized Arabidopsis MAMP receptors are FLS2, which perceives a conserved 22-amino acid peptide (flg22) from bacterial flagellin, and EFR, which

Corresponding author: Libo Shan; E-mail: 1shan@tamu.edu; Telephone: +1.979.845.8818; Fax: +1.979.862.4790.

* The $\boldsymbol{e}$-Xtra logo stands for "electronic extra" and indicates that two supplementary figures are published online and Figure 5 appears in color online. recognizes bacterial elongation factor EF-Tu (Gomez-Gomez and Boller 2000; Zipfel et al. 2006). Upon flagellin perception, FLS2 associates with BAK1, another RLK with a short extracellular LRR domain (Chinchilla et al. 2007; Heese et al 2007). BAK1 was originally identified as a BRI1-associated receptor kinase mediating plant hormone brassinosteroid signaling (Li et al. 2002; Nam and Li 2002). FLS2-BAK1 heteromerization occurs almost instantaneously upon flg22 perception, which likely leads to the subsequent phosphorylation events of FLS2 and BAK1 (Schulze et al. 2010). Significantly, BAK1 is functionally involved in multiple MAMP signaling, and BAK1 heteromerizes with several LRR-RLK, including BRI1, FLS2, and EFR, instantaneously after ligand binding (Schulze et al. 2010). Flg22 also rapidly induces the FLS2and BAK1-dependent phosphorylation of a receptor-like cytoplasmic kinase BIK1 within a minute of signal perception $(\mathrm{Lu}$ et al. 2010; Zhang et al. 2010). BIK1 forms a complex with FLS2/BAK1 in the plasma membrane and transphosphorylates the FLS2/BAK1 receptor complex ( $\mathrm{Lu}$ et al. 2010). Downstream intracellular signaling events in PTI include changes in cytoplasmic $\mathrm{Ca}^{2+}$ levels, activation of mitogen-activated protein kinase (MAPK) cascades, induction of defense-related genes, production of reactive oxygen species, deposition of callose to reinforce the cell wall, and stomatal closure to prevent pathogen entry (Ausubel 2005; Boller and Felix 2009).

To achieve a successful infection, host-adapted pathogens acquired the ability to interfere with plant immunity. Many bacterial effectors secreted through the type III secretion system (TTSS) have been shown to sabotage plant innate immunity. Great strides are being made in the understanding of the biological and enzymatic functions of individual effectors in the past few years, with the development of new technologies and more sensitive and quantitative assays (Block et al. 2008; Gohre and Robatzek 2008; Hann et al. 2010; Lewis et al. 2009; Speth et al. 2007). The Peudomonas syringae effector HopU1, a mono-ADP-ribosyltransferase (ADP-RT), modifies several Arabidopsis RNA-binding proteins, which represent a novel class of ADP-RT substrates (Fu et al. 2007). HopM1, another $P$. syringae effector, targets and degrades a member of the ARF family of guanine nucleotide exchange factors involved in vesicle trafficking (Nomura et al. 2006). We and several other groups discovered that two sequence-distinct but functionally related effectors, AvrPto and AvrPtoB, are potent suppressors of multiple MAMP signaling by targeting MAMP receptor complexes (de Torres et al. 2006; Gimenez-Ibanez et al. 2009; Gohre et al. 2008; Hann and Rathjen 2007; He et al. 2006; Shan et al. 2008; Xiang et al. 2008). In addition to effectors 
from $P$. syringae, DspA/E from Erwinia amylovora interacts with several RLK (Meng et al. 2006), and XopN from Xanthomonas interacts with a tomato atypical RLK and suppresses PTI (Kim et al. 2009). A viral effector protein has also been reported to interact with RLK (Fontes et al. 2004). Notably, the contribution of individual effectors to virulence is difficult to observe with bacterial deletion mutants, likely due to the potential functional redundancy. It is not clear how these effectors cooperatively suppress host immune signaling in plant cells.

Although AvrPto and AvrPtoB are potent suppressors of plant PTI, deletion of AvrPto and AvrPtoB from P. syringae pv. tomato DC3000 only partially reduced its suppression activity on MAMP marker gene expression, suggesting one or more additional effectors exhibiting redundant functions with AvrPto and AvrPtoB in suppressing PTI (He et al. 2006). We identified another DC3000 effector, HopF2, that possesses similar function with AvrPto in suppressing plant PTI. HopF2 is a homolog of $P$. syringae pv. phaseolicola effector AvrPphF, which contributes to distinct avirulence and virulence functions in different bean and soybean cultivars (Jackson et al., 1999; Shan et al. 2004; Tsiamis et al. 2000). HopF2 from DC3000 possesses a putative myristoylation site that is important for its membrane localization and avirulence and virulence functions in tobacco and tomato (Robert-Seilaniantz et al. 2006). In Arabidopsis, HopF2 can block basal resistance, as measured by increased vascular flow (Oh and Collmer 2005), flg22-induced $\mathrm{NHO1}$ induction ( $\mathrm{Li}$ et al. 2005), and callose deposition (Guo et al. 2009), and HopA1-induced cell death (Jamir et al. 2004).

Recently, it has been shown that HopF2 targets RIN4, an important component in both PTI and effector-triggered immunity (ETI) (Wilton et al. 2010). HopF2 interfered with AvrRpt2induced RIN4 degradation, thereby suppressing ETI mediated by AvrRpt2. HopF2 also targeted MAPK kinase 5 (MKK5) and likely other MKK to inhibit PTI signaling in Arabidopsis (Wang et al. 2010). HopF2 possesses ADP-RT activity and ADP-ribosylated MKK5 in vitro. Interestingly, RIN4 was also able to be ADP-ribosylated by HopF2 (Wang et al. 2010). We report here that HopF2 suppresses diverse immune signaling triggered by multiple MAMPs at an immediately early step in PTI signaling. Importantly, HopF2 suppresses flg22-mediated BIK1 phosphorylation, an event upstream of the MAPK cascade in PTI signaling. Expression of HopF2 in transgenic Arabidopsis compromised plant immunity to a nonadaptive bacterium and a necrotrophic fungus. The putative myristoylation site of HopF2 is required for its MAMP suppression function.

\section{RESULTS}

HopF2 intercepts immune signaling activated by multiple MAMPs.

We have shown before that virulent bacteria DC3000 but not its TTSS mutant, hrcC, suppresses MAMP-specific early de-
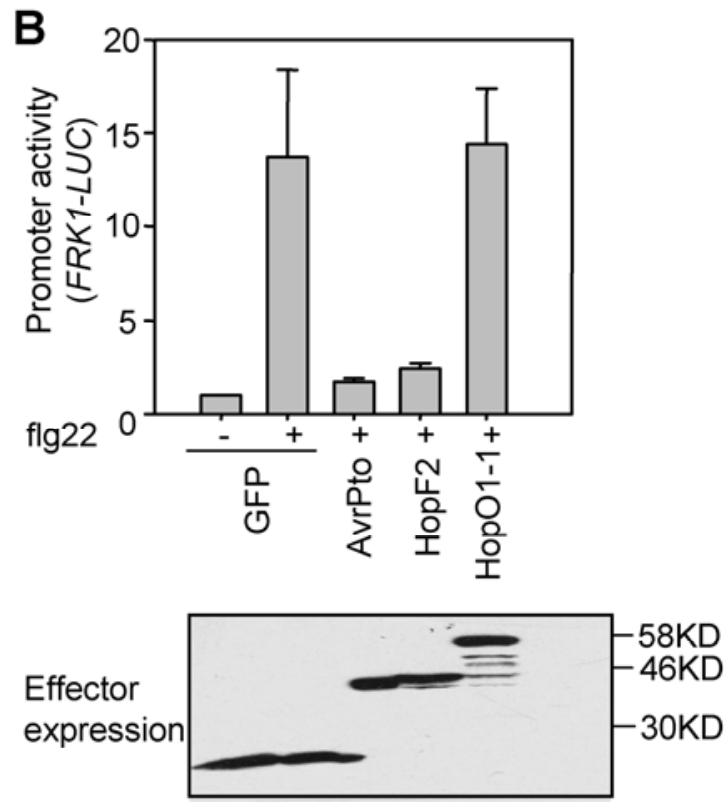

B
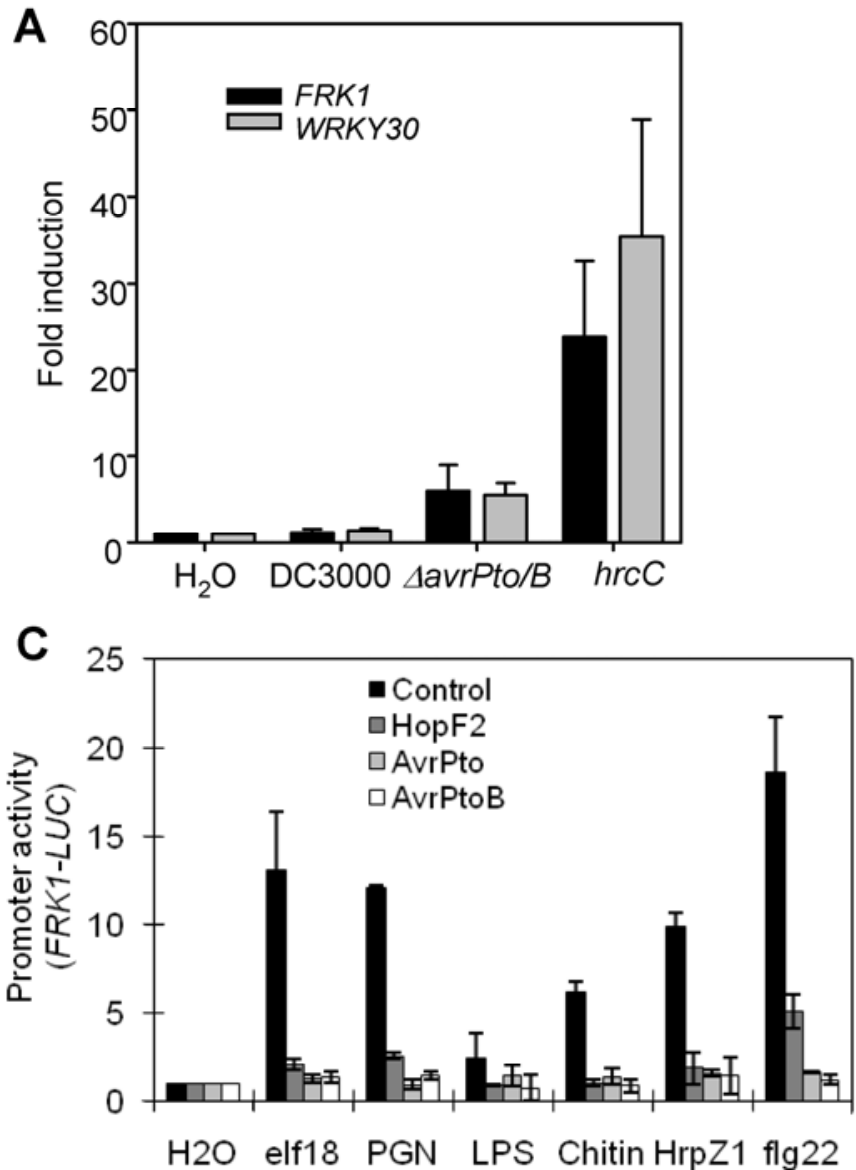

Fig. 1. HopF2 suppresses immune signaling triggered by multiple microbe-associated molecular patterns (MAMPs). A, Real-time reverse transcription-polymerase chain reaction analysis of FRK1 and WRKY30 induction. Four-week-old Arabidopsis plants were inoculated with $\mathrm{H}_{2} \mathrm{O}$, DC3000, DC3000 $\Delta a v r P t o / a v r P t o B$, or DC3000hrcC at $1 \times 10^{8} \mathrm{CFU} / \mathrm{ml}$. The samples were collected $6 \mathrm{~h}$ later for RNA isolation. The gene induction (fold change) by bacterial infiltration was compared with the expression level of $\mathrm{H}_{2} \mathrm{O}$ infiltration. B, HopF2 suppresses flg22-induced FRK1-LUC activation. Protoplasts were cotransfected with a green fluorescent protein (GFP) control or an effector and $F R K 1-L U C$ reporter. Three hours later, transfected protoplasts were treated with $10 \mathrm{nM}$ flg22 for another $3 \mathrm{~h}$. The expression of effectors was detected by anti-GFP Western blot. C, HopF2 suppresses multiple MAMP-mediated FRK1-LUC activation. Protoplasts were transfected with FRK1-LUC with or without HopF2, AvrPto, or AvrPtoB for different MAMP treatments. The concentrations for different MAMPs are flg22 = $10 \mathrm{nM}$, elf18 = $10 \mathrm{nM}, \mathrm{HrpZ1}=100 \mathrm{nM}$, peptidoglycan $(\mathrm{PGN})=50 \mu \mathrm{g} / \mathrm{ml}$, chitin $=50 \mu \mathrm{g} / \mathrm{ml}$, and lipopolysaccharide $(\mathrm{LPS})=50 \mu \mathrm{g} / \mathrm{ml}$. 
fense-gene activation (He et al. 2006). A cell-based genetic screen has identified AvrPto and AvrPtoB as potent suppressors of early MAMP signaling. However, the DC3000_avrPto $\triangle a v r P t o B$ double mutant only partially reduced the suppression activity of DC3000 on MAMP marker gene expression (Fig. 1A). As shown by quantitative reverse transcription-polymerase chain reaction (RT-PCR) analysis, DC3000 hrcC strongly activated the expression of two early MAMP marker genes, FRK1 and WRKY30, in Arabidopsis plants $6 \mathrm{~h}$ after infiltration. The induction of these genes was not observed by DC3000, due to the collective suppression activity of type III effectors. Deletion of avrPto and avrPtoB from DC3000 partially restored $F R K 1$ and $W R K Y 30$ expression but to a much lesser extent than DC3000 hrcC. The data suggest that additional effectors likely exist in the suppression of early MAMP signaling.

We cloned several additional bacterial effectors tagged with green fluorescent protein (GFP) in a plant expression vector and determined their effect on the flg22 activation of the FRK1-LUC reporter with an Arabidopsis protoplast transient assay. Significantly, HopF2 but not HopO1-1 suppressed flg22 activation of FRK1-LUC. The effect of HopF2 on suppressing flg22-mediated gene transcription was almost as potent as AvrPto (Fig. 1B). The data also confirmed the report by Wang and associates (2010) about the suppression of flg22-induced FRK 1 by HopF2. Plants respond to multiple MAMPs and activate the convergent signaling. To determine whether HopF2 interrupts the immune response activated by other MAMPs in addition to flg22, we treated HopF2 transfected protoplasts with elf18 (the 18-amino acid peptide of EF-Tu), peptidoglycan, lipopolysaccharide, HrpZ1 harpin, and chitin. Similar to AvrPto and AvrPtoB, HopF2 dramatically inhibited the activation of FRK1 promoter by all these MAMPs (Fig. 1C). The results indicate that HopF2 suppresses the immune signaling triggered by multiple MAMPs and likely targets to a convergent component in plant innate immunity.

\section{HopF2 attenuates MAPK signaling.}

MAPK activation is a convergent early-signaling event activated by different MAMPs in plants. It has been reported that flg22 activates MPK3, MPK4, and MPK6 in Arabidopsis (Asai et al. 2002; Rodriguez et al. 2010; Suarez-Rodriguez et al. 2007). To determine whether HopF2 suppresses the flg22 activation of MAPK, we performed an immunocomplex kinase assay. Arabidopsis protoplasts were cotransfected with hemagglutinin (HA)-tagged MAPK and GFP-tagged effectors. The MAPK activation was stimulated with flg22 treatment for 10 min. Consistent with the previous report, expression of AvrPtoB completely blocked the activation of MPK3 and MPK6 by flg22 (Fig. 2A). Similarly, expression of HopF2 in protoplasts significantly diminished the flg22 activation of MPK3 and MPK6. In addition, both AvrPtoB and HopF2 suppressed the activation of MPK4 by flg22 (Fig. 2A). These results confirmed the recent report by Wang and associates (2010). The activation of MPK3 and MPK6 by elf18 was also largely abolished by the expression of HopF2 (Fig. 2B). The data indicate that HopF2 suppresses MAMP-induced early gene transcription and MAPK activation.

\section{HopF2 intercepts flg22-induced BIK1 phosphorylation.}

We recently showed that flg22 rapidly induces a cytoplasmic kinase BIK1 phosphorylation within a minute of signal perception (Lu et al. 2010). BIK1 is a plasma membrane-localized protein forming a complex with FLS2/BAK1(Lu et al. 2010; Veronese et al. 2006). Significantly, flg22-induced BIK1 phosphorylation was suppressed by expression of HopF2 in

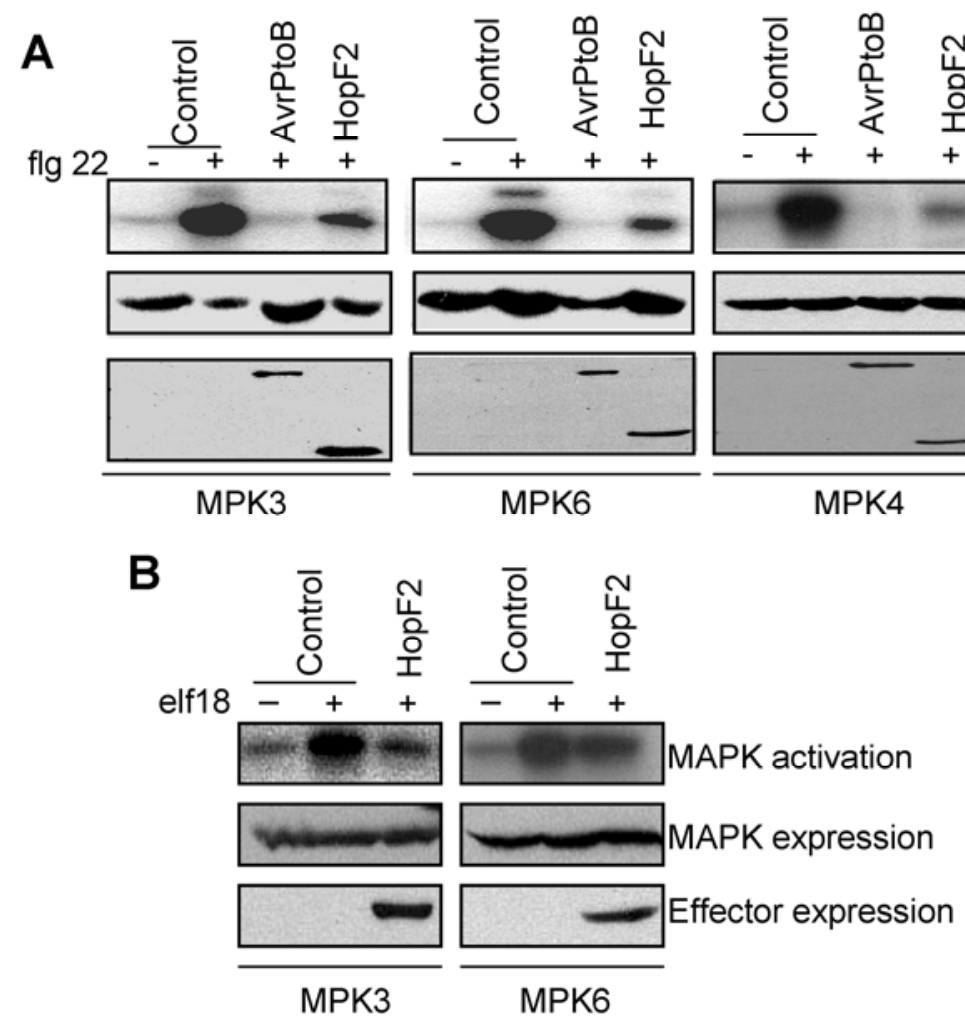

Fig. 2. HopF2 attenuates mitogen-activated protein kinase (MAPK) signaling. A, HopF2 suppresses flg22-mediated MPK3, MPK4, and MPK6 activation. hemagglutinin (HA)-tagged MPK3, MPK4, or MPK6 was coexpressed with green fluorescent protein-tagged effectors. Transfected protoplasts were incubated for $6 \mathrm{~h}$ before $1-\mu \mathrm{M}$ flg22 treatment for $10 \mathrm{~min}$. An anti-HA antibody was used for immunoprecipitation of MAPK. Kinase activity was detected by an in-vitro kinase assay (top). Protein expression is shown for MAPK (middle) and effectors (bottom). B, HopF2 suppresses elf18-mediated MPK3 and MPK6 activation. Transfected protoplasts were treated with $1 \mu \mathrm{M}$ elf 18 for $10 \mathrm{~min}$. 
protoplasts (Fig. 3A). BIK1 phosphorylation was indicated as a mobility shift upon flg22 stimulation. This mobility shift was not observed when BIK1 was coexpressed with HopF2 in protoplasts (Fig. 3A). Expression of AvrPto also completely blocked BIK1 phosphorylation. The data are consistent with the plasma-membrane localization of HopF2 and suggest that HopF2 suppresses MAMP-mediated signaling at an immediately early step. We further tested whether HopF2 could, singly or together, potentially target or de-phosphorylate BIK1 phosphorylation in vitro. HopF2 did not interact with BIK1 in a coimmunoprecipitation assay (Supplementary Fig. S1) or a yeast two-hybrid assay (data not shown). Furthermore, HopF2 did not affect BIK1 autophosphorylation and its phosphorylation on BAK1with an in vitro kinase assay (Fig. 3B), indicat-

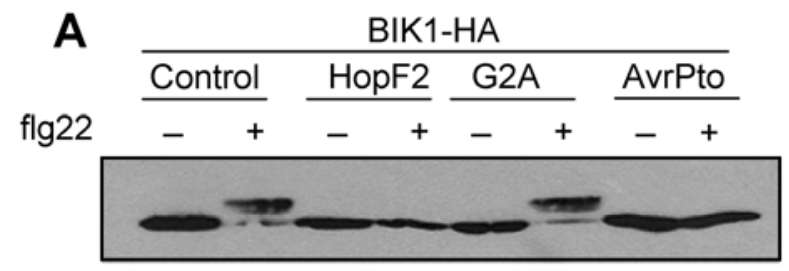

BIK1-HA expression

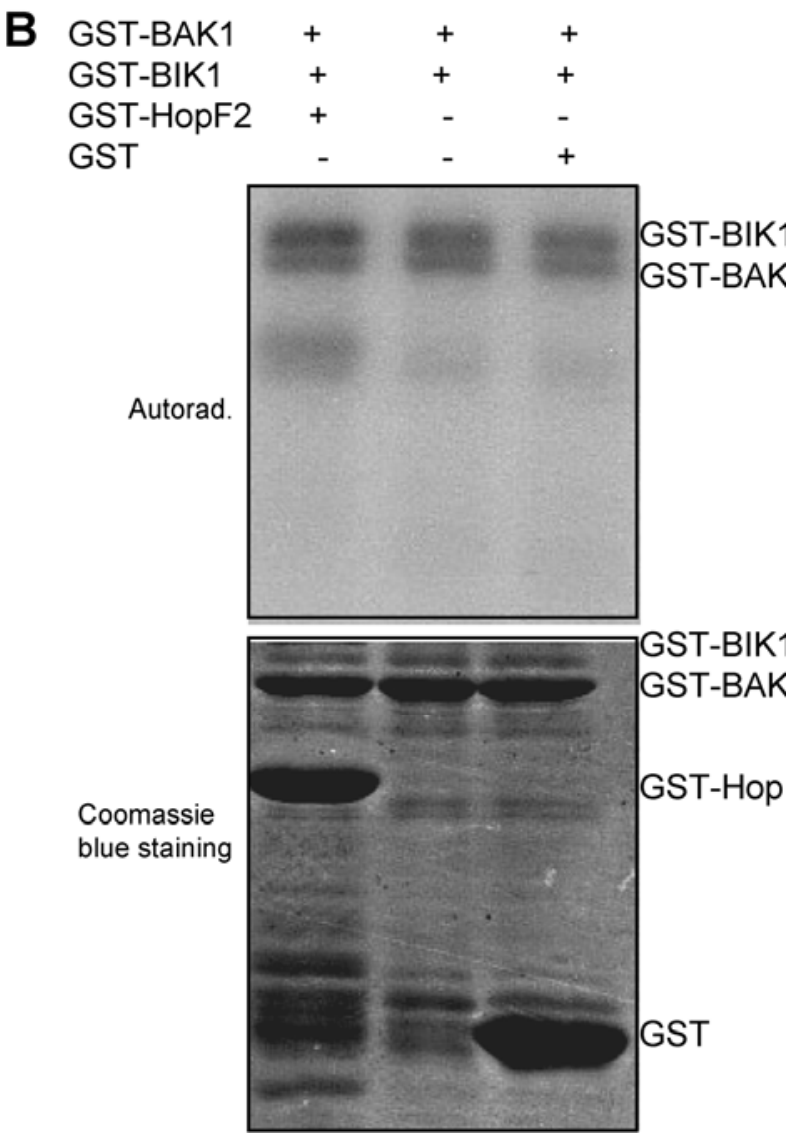

Fig. 3. HopF2 suppresses flg22-induced BIK1 phosphorylation in vivo. A, HopF2 blocks flg22-induced BIK1 phosphorylation. Protoplasts were cotransfected with BIK1-HA (hemagglutinin) and green fluorescent proteintagged HopF2, HopF2G2A, or AvrPto for $6 \mathrm{~h}$ and were treated with $1 \mu \mathrm{M}$ flg22 for 10 min. B, HopF2 did not affect BIK1 autophosphorylation and phosphorylation on BAK1. An in vitro kinase assay was performed by incubating GST-BIK1 and GST-BAK1K with or without GST-HopF2. Proteins were separated with sodium dodecyl sulfate-polyacrylamide gel electrophoresis and were analyzed by autoradiography (top panel). The top band is autophosphorylated GST-BIK1, and then, phosphorylated GSTBAK1K. The protein loading control was shown by Coomassie blue staining (bottom panel). ing that HopF2 does not directly target and inhibit BIK1 kinase activity.

\section{AvrPphC does not affect}

HopF2 MAMP suppression function in Arabidopsis.

In some bean cultivars, the effector AvrPphC from $P$. syringae pv. phaseolicola suppresses the AvrPphF avirulence function mediated by the $\mathrm{R} 1$ resistance protein (Tsiamis et al. 2000). We tested whether AvrPphC could interfere with HopF2 MAMP suppression function. We coexpressed HopF2 and AvrPphC and tested the activation of FRK1-LUC upon flg22 treatment in protoplasts. As expected, HopF2 significantly suppressed flg22-induced FRK1-LUC activation. AvrPphC itself had no effect on FRK1-LUC induction. The expression of AvrPphC did not ameliorate the strong suppression by HopF2 on FRK1-LUC induction (Supplementary Fig. S2), suggesting that AvrPphC did not affect HopF2 virulence in Arabidopsis.

\section{Myristoylation and conserved surface residues of HopF2 are essential for its MAMP suppression activity.}

HopF2 has a putative myristoylation site at its N-terminus, and mutation of this site disrupts its membrane localization (Robert-Seilaniantz et al. 2006). More importantly, the putative myristoylation site is required for HopF2 avirulence and virulence functions in tobacco and tomato. We examined whether the putative myristoylation site is essential for HopF2 suppression function in MAMP signaling. We substituted the putative myristoylation site Gly with Ala (G2A) and tested its effect on flg22-induced FRK1-LUC activation. As shown in Figure 4A, HopF2 G2A mutation was unable to suppress flg22 induction of FRK1-LUC. In addition, HopF2 G2A also lost the ability to block flg22-mediated BIK1 phosphorylation (Fig. $3 \mathrm{~A})$. Taken together, the data suggest that plasma membrane localization of HopF2 is required for its MAMP suppression activity and HopF2 likely targets to a membrane-associated component for its MAMP suppression function.

Structural analysis of AvrPphF identified two clusters of conserved surface-exposed residues based on certain structural similarity to the catalytic domain of multiple bacterial ADPRT (Singer et al. 2004). Mutational analysis of AvrPphF indicated that R72 and D174 in pocket A are required for its virulence function in susceptible bean cultivar Tendergreen and its avirulence function in resistant cultivar Red Mexican (Singer et al. 2004). S90 in pocket A and $\mathrm{H} 97$ and $\mathrm{E} 98$ in pocket $\mathrm{B}$ are less essential for AvrPphF virulence and avirulence functions. We individually substituted the corresponding residues in HopF2 with Ala and tested its ability to suppress flg22 activation of FRK1-LUC. Significantly, the R71A and D175A mutations of HopF2 in pocket A, which are the equivalent of R72 and D174 in AvrPphF, completely lost the ability to suppress flg22 activation of FRK1-LUC (Fig. 4A), confirming the recent report by Wang and associates (2010). The mutations in S89, H96, and E97 of HopF2 had little or no effect on its MAMP suppression activity. We further tested whether R71 and D175 are required for HopF2 to suppress flg22-induced MAPK activation with an immunocomplex kinase assay. As shown in Figure 4B, although wild-type HopF2 dramatically suppressed flg22-activated MPK3 and MPK4, HopF2 R71A and D175A were no longer able to suppress MPK3 and MPK4 activation by flg22. H96A still kept the ability to block MAPK activation. The protein expression levels of different HopF2 mutants were comparable to that of wild-type HopF2 (Fig. 4B).

\section{HopF2 is a suppressor of plant nonhost immunity.}

Plant nonhost immunity is, in part, attributed to plant PTI (Ham et al. 2007; He et al. 2006; Li et al. 2005). To test whether HopF2 could compromise plant nonhost immunity, 
we generated HopF2 transgenic plants under the control of the dexamethasone-inducible promoter. We examined the growth of a nonadaptive bacterial strain $P$. syringae pv. phaseolicola in wild-type plants and transgenic plants expressing HopF2. Three days after infection, bacterial populations in three independent lines of HopF2 transgenic plants were about five- to 10-fold higher than that in wild-type control plants (Fig. 5A). The susceptibility of HopF2 transgenic plants to $P$. syringae pv. phaseolicola infection was comparable to that of AvrPto transgenic plants (Fig. 5A).

Consistent with transient expression of HopF2 in protoplasts, the expression of HopF2 in stable transgenic plants suppressed flg22-activation of endogenous MAMP marker genes by RT-PCR analysis (Fig. 5B). FRK1, WRKY30, and At3g23230 were strongly induced 0.5 and $1 \mathrm{~h}$ after flg 22 treatment in control plants, while their induction was largely diminished in HopF2 transgenic plants (Fig. 5B). Expression of HopF2 in plant cells suppressed the immune responses triggered by MAMPs from both bacteria and fungi. We found that HopF2 transgenic plants were more susceptible to a necrotrophic fungal pathogen, Botrytis cinerea, than wild-type control plants (Fig. 5C and D). Compared with wild-type plants, HopF2 transgenic plants showed increased disease symptoms with chlorosis and necrosis (Fig. 5C) and enlarged infection areas (Fig. 5D) after Botrytis cinerea infection. The similar results were observed with AvrPto transgenic plants (Fig. 5C and D).

\section{DISCUSSION}

To combat the pathogenic invaders, plants have evolved strategies and tactics to instantaneously recognize an array of MAMPs and launch a complex network of immune signaling. We report here that $P$. syringae effector HopF2 potently suppresses diverse immune signaling events triggered by conserved microbial components of bacteria and fungi. The suppression apparently occurs at a very early and essential step immediately upon MAMP perception on the plasma membrane. HopF2 is a membrane-localized protein with a putative myristoylation site (Robert-Seilaniantz et al. 2006). The membrane association is required for its MAMP suppression function. Consistently, HopF2 intercepts flg22-mediated phosphorylation of BIK1, a plasma membrane-localized kinase interacting with the FLS2/BAK1 complex (Lu et al. 2010; Zhang et al. 2010). Apparently, HopF2 does not directly interact with BIK1 or inhibit BIK1 kinase activity (Fig. 3B). BAK1, likely functioning upstream of BIK1 (Lu et al. 2010), is required for multiple MAMP responses and appears to function in distinct receptorsignaling complexes to integrate MAMP perception into downstream signaling events (Chinchilla et al. 2007; Heese et al. 2007; Schulze et al. 2010). It is likely that HopF2 also targets BAK1 or other convergent components residing in the plasma membrane in transducing MAMP signaling.

Host-adapted pathogens employ various means to dampen host immunity. $P$. syringae injects about 30 virulence effectors into plant cells (Lindeberg et al. 2006, 2009). These effectors deploy diverse biochemical activities to jeopardize various plant cellular processes. Many elegant studies have demonstrated a general pattern in which a single effector targets multiple host factors at critical steps in key host processes and have shown distinct effectors can converge on a specific host target or cellular compartment to achieve the virulence robustness and redundancy (Hann and Rathjen 2010; Lewis et al. 2009). It remains a challenge to address how the discrete activities of these effectors are precisely coordinated and temporally controlled. Several Salmonella effectors (SipA, SipC, SopB, SopE, SopE2, and SptP) and translocase SipB are all delivered to the cell plasma membrane, suggesting that the plasma membrane is a critical interface for effector-effector interplay and
A

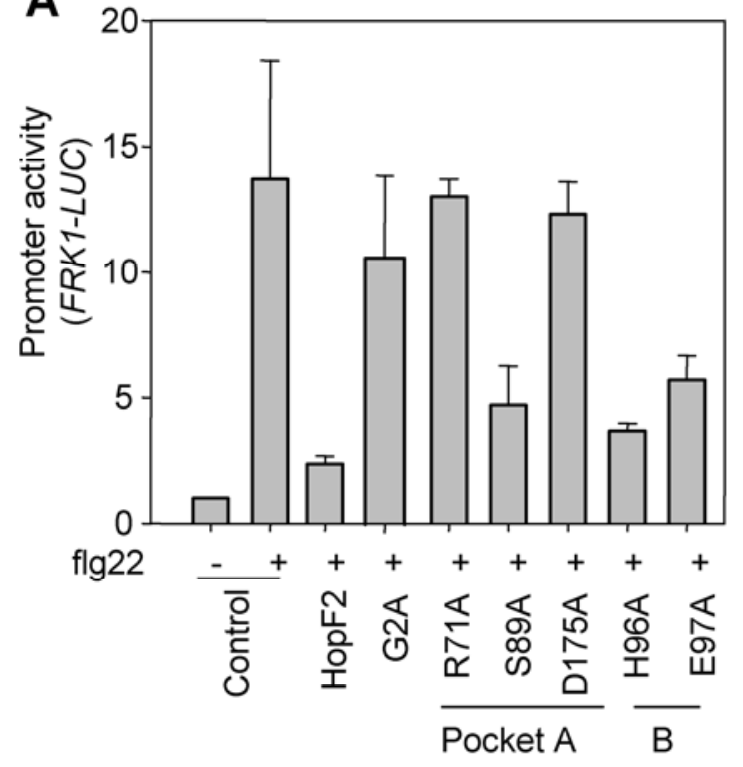

B
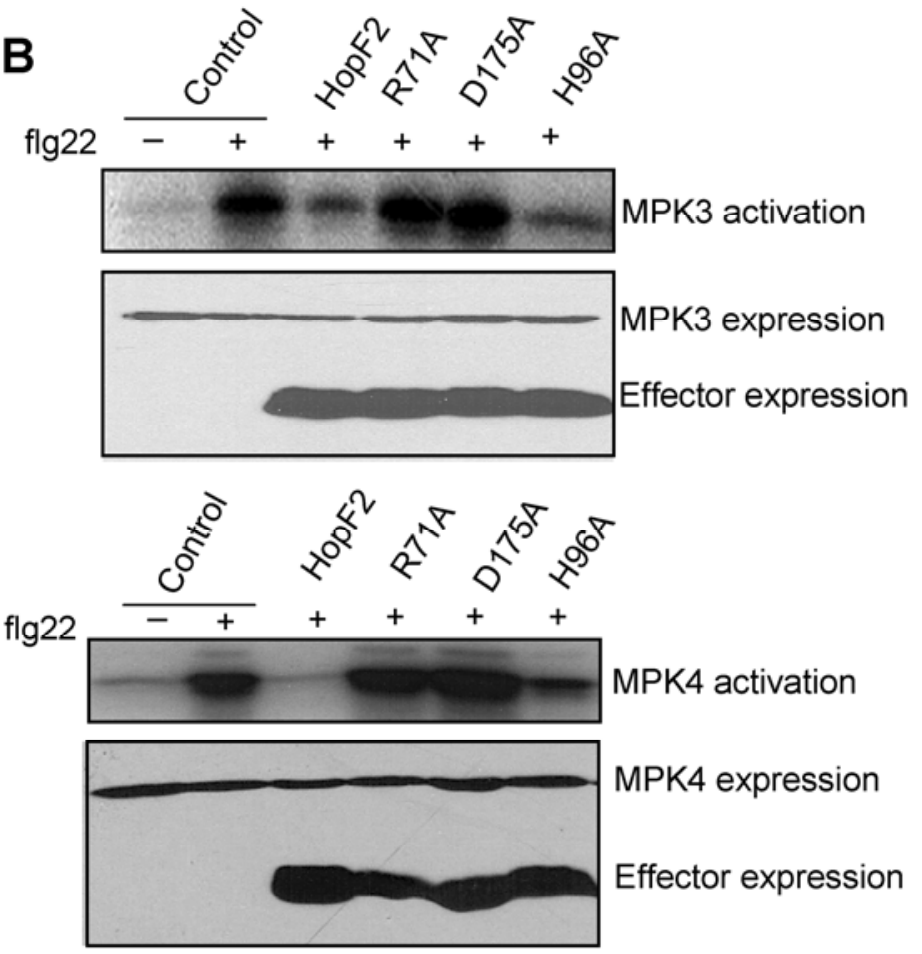

Fig. 4. Myristoylation and conserved surface residues of HopF2 are required for its mitogen-activated molecular pattern (MAMP)-suppression function. A, G2, R71, and D175 are required for HopF2 suppression of FRK1 induction. Protoplasts were cotransfected with HopF2 or its mutants and FRK1-LUC reporter and were incubated for $3 \mathrm{~h}$ before treatment with $10 \mathrm{nM}$ flg22 for another $3 \mathrm{~h}$. B, R71 and D175 are required for HopF2 suppression of mitogenactivated protein kinase (MAPK) activation. Hemagglutinin (HA)-tagged MAPK was coexpressed with HA-tagged HopF2 or its mutants for 6 h before 1$\mu \mathrm{M}$ flg22 treatment for $10 \mathrm{~min}$. Kinase activity was detected by an in vitro kinase assay (top). Protein expression is shown for MAPK and effectors (bottom). 
effector-target interaction during bacterial entry (Cain et al. 2004; McGhie et al. 2009; Raffatellu et al. 2005). Sophisticated functional interactions between these Salmonella effectors following their delivery into the host cells were revealed by a systemic experimental screen (Cain et al. 2008). Localization studies suggest that several $P$. syringae effectors localize to the plasma membrane in plant cells through the putative myristoylation site (Nimchuk et al. 2000; Robert-Seilaniantz et al. 2006; Shan et al. 2000), implicating the plant plasma membrane as a battlefield for coordinated effector actions to promote bacterial invasion. Indeed, several plasma membrane-localized effectors, including HopF2, AvrPto, and AvrRpm1, are potent suppressors of PTI signaling (He et al. 2006; Kim et al. 2005; Wang et al. 2010) It is of great interest to discover how these effectors work in harmony in suppressing plant immune signaling under natural infection conditions.

Recently, HopF2 has been shown to directly interact with Arabidopsis RIN4, a virulence target of three additional $P$. syringae effectors, AvrRpm1, AvrRpt2, and AvrB (Wilton et al. 2010). RIN4 is a plasma membrane-localized protein interacting with two resistance proteins RPM1 and RPS2 (Axtell and Staskawicz 2003; Liu et al. 2009; Mackey et al. 2002, 2003).
RIN4 is phosphorylated upon infection by bacteria carrying AvrRpm1 or AvrB, whereas RIN4 is cleaved by AvrRpt 2 to induce ETI. Expression of HopF2 in transgenic plants suppressed AvrRpt2-induced RIN4 cleavage and AvrRpt2-mediated ETI (Wilton et al. 2010). Importantly, HopF2 promoted $P$. syringae growth in a RIN4-dependent manner, suggesting that RIN4 is a virulence target of HopF2 in Arabidopsis (Wilton et al. 2010). Apparently, targeting RIN4 does not count for the full virulence function of HopF2, since it has not been reported that RIN4 is deficient in flg22-induced MAPK activation and early gene transcription. Instead, RIN4 has been proposed to be a negative regulator of PTI (Kim et al. 2005; Mackey et al. 2002).

While this manuscript was being prepared, Wang and associates (2010) reported that HopF2 inhibited flg22 signaling by targeting MKK5 and other MKK. HopF2 ADP-ribosylates MKK5 in vitro (Wang et al. 2010). HopF2 is a membranelocalized protein and membrane association is required for its MAMP suppression and virulence and avirulence functions in different plant species. It remains unknown where MKK localize and how HopF2 interacts with diverse MKK in plant cells. Apparently, flg22 signaling constitutes MAPK-dependent and
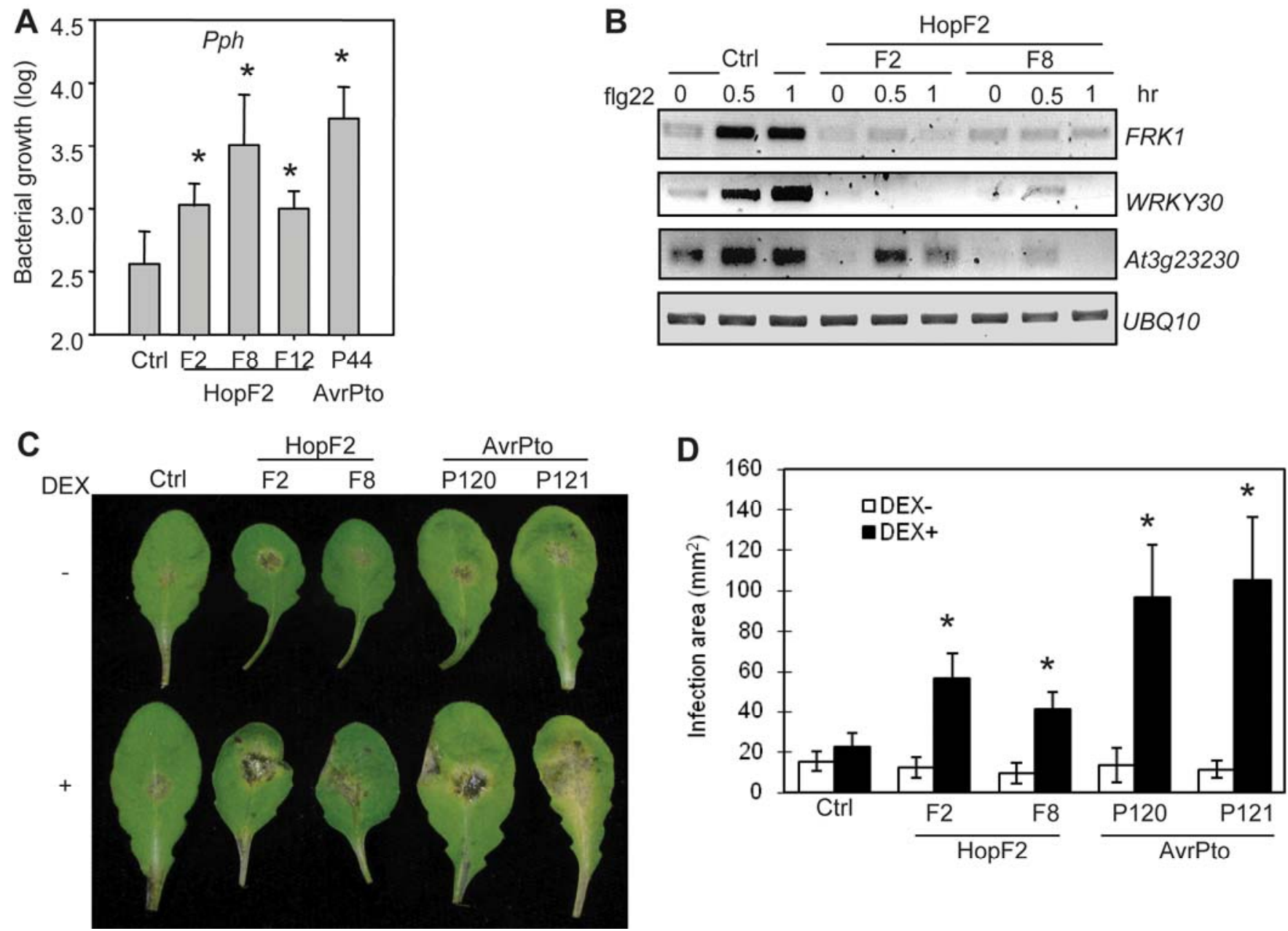

Fig. 5. HopF2 suppresses plant nonhost resistance. A, HopF2 transgenic plants support nonadaptive bacteria growth. Four-week-old plants (Col-0 control, HopF2, or AvrPto transgenic plants) were sprayed with $10 \mu \mathrm{M}$ dexamethasone (DEX) for $24 \mathrm{~h}$ before bacterial inoculation. Arabidopsis leaves were inoculated with Pseudomonas syringae pv. phaseolicola NPS3121 (Pph) at $5 \times 10^{5} \mathrm{CFU} / \mathrm{ml}$. The bacterial counting was performed 3 days after inoculation. An asterisk (*) indicates a significant difference with $P<0.05$ when compared with data from control plants, based on the results of an unpaired Student's $t$-test. B, Reverse transcription-polymerase chain reaction analysis of microbe-associated molecular pattern marker-gene induction. Ten-day-old seedlings were treated with $10 \mu \mathrm{M}$ DEX for $24 \mathrm{~h}$ and were then treated with $10 \mathrm{nM}$ flg22 for 0.5 and $1 \mathrm{~h}$. C, Susceptibility to Botrytis cinerea. Four-week-old plants were sprayed with $10 \mu \mathrm{M}$ DEX for $24 \mathrm{~h}$ before Botrytis cinerea BO5 was sprayed at a concentration of $10^{5}$ spores per milliliter. Disease symptoms were recorded 2 days after infection. D, Lesion development of $B$. cinerea infection. The area of infection was measured 4 days postinoculation. An asterisk $(*)$ indicates a significant difference with $P<0.05$. 
-independent pathways (Asai et al. 2002; Boudsocq et al. 2010). Given the potent virulence function of HopF2 in suppressing multiple MAMP signaling, it is likely that HopF2 targets both MAPK-dependent and -independent pathways or a convergent upstream component immediately after MAMP perception. Consistent with this, expression of HopF2 inhibited flg22-mediated phosphorylation of BIK1, which is phosphorylated within a minute of flg22 perception. Accumulating evidence suggests that BIK1 likely functions upstream or independent of the MAPK cascade in plant innate immunity (Lu et al. 2010; Veronese et al. 2006; Zhang et al. 2010). In particular, it has been shown that the MKK inhibitor did not suppress flg22induced BIK1 phosphorylation and active MKK5 did not phosphorylate BIK1, suggesting that MKK5 is not required for BIK1 phosphorylation (Lu et al. 2010). Thus, our data imply that HopF2 targets a plasma membrane-localized component upstream of BIK1 phosphorylation and the MAPK cascade in PTI signaling. Together with the data by Wang and associates (2010) and Wilton and associates (2010), it appears that HopF2 targets mutiple host factors in suppressing plant PTI and ETI signaling.

\section{MATERIALS AND METHODS}

\section{Plant growth and pathogen assays.}

Arabidopsis plants were grown in a growth chamber at $23^{\circ} \mathrm{C}$ with a 13-h photoperiod for 30 days before RNA isolation, bacterial inoculation, or protoplast isolation. Different $P$. syringae strains were grown overnight at $28^{\circ} \mathrm{C}$ in King's B (KB) medium with appropriate antibiotics, with final concentration of antibiotics as follows (in milligrams per liter): kanamycin (50), tetracycline (20, streptomycin (100), spectinomycin (50), and gentamycin (10). Bacteria were pelleted by centrifugation, were washed, and were diluted to the desired density with $10 \mathrm{mM}$ $\mathrm{MgCl}_{2}$. Arabidopsis leaves were infiltrated with bacteria using a needleless syringe, for RNA isolation and bacterial counting. To measure bacterial growth, two leaf disks were ground in $100 \mu \mathrm{l}$ of $\mathrm{H}_{2} \mathrm{O}$, and serial dilutions were plated on $\mathrm{KB}$ medium with the appropriate antibiotic. Bacterial colony forming units were counted 2 days after incubation at $28^{\circ} \mathrm{C}$. Each datapoint is shown as triplicates.

Botrytis cinerea BO5 was cultured on potato dextrose agar (Difco, Detroit) and was incubated at room temperature. Conidia were resuspended in distilled water and the spore concentration was adjusted to $10^{5}$ spores per milliliter. Gelatin $(0.5 \%)$ was added to conidial suspension before inoculation. Spores were sprayed onto whole Arabidopsis plants, and disease development was monitored over a period of 5 days.

\section{Generation of HopF2 transgenic plants.}

HopF2 transgenic plants were generated by Agrobacteriummediated transformation with the HopF2 construct under the control of the dexamethasone-inducible promoter with an HA epitope tag (McNellis et al., 1998). To induce HopF2 expression, transgenic plants were sprayed with $10 \mu \mathrm{M}$ dexamethasone containing $0.025 \%$ Silwet L-77 1 day before infiltration with flg22 or bacteria for RT-PCR and disease assays.

\section{Plasmid constructs and point mutations.}

The effector constructs were made by cloning PCR fragments from $P$. syringae pv. tomato DC 3000 genomic DNA into a plant expression vector with a GFP tag at the $\mathrm{C}$ terminus. HopF2 was amplified with primers 5'-CGGGATCCATGGGT AATATTTGCGGCACC-3' and 5'-GAAGGCCTGACCCTTT CGACCGGCACTTTC-3', in which the ATA start codon was replaced with ATG; HopO1-1 with 5'-CGGGATCCATGGGT AATATTTGTGGTAC-3' and 5'-GAAGGCCTCTCGTCAGA
GCTCTCTGC-3'; AvrPphC with 5'-CATGCCATGGGAAAT GTTTGTTTCCG-3' and 5'-GAAGGCCTCTGAGGGGGCCG CTCAAAAAG- $3^{\prime}$. The other effector, reporter, and MAPK constructs were reported previously (He et al. 2006).

Point mutations were generated by the site-specific mutagenesis kit (Stratagene, La Jolla, CA, U.S.A.) using the following primers: 5'-CGGGATCCATGGCTAATATTTGCGGC ACC- $3^{\prime}$ and $5^{\prime}$-GGTGCCGCAAATATTAGCCATGGATCCC G-3'; R71A with 5'-GATACCGAGCTTTTCGCAACGACGG ATAGTCGC-3' and 5'-GCGACTATCCGTCGTTGCGAAAA GCTCGGTATC-3'; S89A with 5'-GCGGGCAATCCACAAG CCATGGCGAGTATCC-3' and 5'-GGATACTCGCCATGGCT TGTGGATTGCCCGC-3'; H96A with 5'-CAGGTGCCCAAC CAGCCGAAGCAAGGGCGTAC-3' and 5'-GTACGCCCTTG CTTCGGCTGGTTGGGCACCTG-3'; E97A with 5'-GTGCC CAACCACACGCAGCAAGGGCGTACG-3' and 5'-CGTACG CCCTTGCTGCGTGTGGTTGGGCAC-3'; D175A with 5'-GC AAGGTCTATGCCGCCGCTTCGTCTGTAGC-3' and $5^{\prime}$-GC TACAGACGAAGCGGCGGCATAGACCTTGC-3’.

\section{Arabidopsis protoplast transient expression and MAPK assays.}

Protoplast transient expression and in vitro MAPK assays were carried out as described previously (He et al. 2006, 2007). UBQ10-GUS was cotransfected with FRK1-LUC as an internal control, and the promoter activity was presented as the LUC/GUS ratio. Protoplasts were collected $6 \mathrm{~h}$ after transfection for protein expression, kinase activity, and promoter activity assays. Protoplasts transfected with plasmid DNA without effectors were used as controls.

\section{RT-PCR analysis.}

Total RNA was isolated from leaves after bacterial or flg22 treatment by using TRIzol Reagent (Invitrogen, San Diego, CA, U.S.A.). Complementary DNA was synthesized from 1.5 $\mu \mathrm{g}$ of total RNA, using $0.1 \mu \mathrm{g}$ oligo(dT) primer and reverse transcriptase (Invitrogen). RT-PCR was run for 35 cycles. Realtime RT-PCR analysis was carried out with an ABI 7900HT sequence detection system (Applied Biosystems, Foster City, CA, U.S.A.). The expression of FRK1, WRKY30, or At3g23230 was normalized to the expression of $U B Q 10$. WRKY30 and At3g23230 were amplified with the primers 5'-GCAGCTTGA GAGCAAGAATG-3' and 5'-AGCCAAATTTCCAAGAGGAT$3^{\prime}$ for WRKY30, and 5'-CTCAGCGGTCTCAAATGTCC-3' and 5'AGGAGCAGCAACAACCAATC-3' for At3g23230. The primers for $F R K 1$ and $U B Q 10$ were reported by He and associates (2006).

\section{ACKNOWLEDGMENTS}

This work was initiated in J. Sheen's laboratory at Massachusetts General Hospital and Harvard Medical School with the support by grant from the National Institute of Health (NIH) (no. R01GM70567) to J. Sheen. Work in the laboratories of L. Shan and P. He was supported by Texas A\&M University start-up funds to L. Shan and P. He, and NIH (no. R01GM092893) to P. He and the National Science Foundation (no. IOS1030250) to L. Shan.

\section{LITERATURE CITED}

Asai, T., Tena, G., Plotnikova, J., Willmann, M. R., Chiu, W. L., GomezGomez, L., Boller, T., Ausubel, F. M., and Sheen, J. 2002. MAP kinase signalling cascade in Arabidopsis innate immunity. Nature 415:977983.

Ausubel, F. M. 2005. Are innate immune signaling pathways in plants and animals conserved? Nat. Immunol. 6:973-979.

Axtell, M. J., and Staskawicz, B. J. 2003. Initiation of RPS2-specified disease resistance in Arabidopsis is coupled to the AvrRpt2-directed elimination of RIN4. Cell 112:369-377. 
Block, A., Li, G., Fu, Z. Q., and Alfano, J. R. 2008. Phytopathogen type III effector weaponry and their plant targets. Curr. Opin. Plant Biol. 11:396-403.

Boller, T., and Felix, G. 2009. A renaissance of elicitors: Perception of microbe-associated molecular patterns and danger signals by patternrecognition receptors. Annu. Rev. Plant Biol. 60:379-406.

Boudsocq, M., Willmann, M. R., McCormack, M., Lee, H., Shan, L., He, P., Bush, J., Cheng, S. H., and Sheen, J. 2010. Differential innate immune signalling via $\mathrm{Ca}^{2+}$ sensor protein kinases. Nature 464:418422

Cain, R. J., Hayward, R. D., and Koronakis, V. 2004. The target cell plasma membrane is a critical interface for Salmonella cell entry effector-host interplay. Mol. Microbiol. 54:887-904.

Cain, R. J., Hayward, R. D., and Koronakis, V. 2008. Deciphering interplay between Salmonella invasion effectors. PLoS Pathog. 4:e1000037. Published online.

Chinchilla, D., Zipfel, C., Robatzek, S., Kemmerling, B., Nurnberger, T., Jones, J. D., Felix, G., and Boller, T. 2007. A flagellin-induced complex of the receptor FLS2 and BAK1 initiates plant defence. Nature 448:497-500

de Torres, M., Mansfield, J. W., Grabov, N., Brown, I. R., Ammouneh, H., Tsiamis, G., Forsyth, A., Robatzek, S., Grant, M., and Boch, J. 2006. Pseudomonas syringae effector AvrPtoB suppresses basal defence in Arabidopsis. Plant J. 47:368-382.

Fontes, E. P., Santos, A. A., Luz, D. F., Waclawovsky, A. J., and Chory, J. 2004. The geminivirus nuclear shuttle protein is a virulence factor that suppresses transmembrane receptor kinase activity. Genes Dev. $18: 2545-2556$

Fu, Z. Q., Guo, M., Jeong, B. R., Tian, F., Elthon, T. E., Cerny, R. L., Staiger, D., and Alfano, J. R. 2007. A type III effector ADP-ribosylates RNA-binding proteins and quells plant immunity. Nature 447:284-288.

Gimenez-Ibanez, S., Hann, D. R., Ntoukakis, V., Petutschnig, E., Lipka, V., and Rathjen, J. P. 2009. AvrPtoB targets the LysM receptor kinase CERK1 to promote bacterial virulence on plants. Curr. Biol. 19:423429

Gohre, V., and Robatzek, S. 2008. Breaking the barriers: microbial effector molecules subvert plant immunity. Annu. Rev. Phytopathol. 46:189215

Gohre, V., Spallek, T., Haweker, H., Mersmann, S., Mentzel, T., Boller, T., de Torres, M., Mansfield, J. W., and Robatzek, S. 2008. Plant patternrecognition receptor FLS2 is directed for degradation by the bacterial ubiquitin ligase AvrPtoB. Curr. Biol. 18:1824-1832.

Gomez-Gomez, L., and Boller, T. 2000. FLS2: An LRR receptor-like kinase involved in the perception of the bacterial elicitor flagellin in Arabidopsis. Mol. Cell 5:1003-1011.

Guo, M., Tian, F., Wamboldt, Y., and Alfano, J. R. 2009. The majority of the type III effector inventory of Pseudomonas syringae pv. tomato DC3000 can suppress plant immunity. Mol. Plant-Microbe Interact. 22:1069-1080.

Ham, J. H., Kim, M. G., Lee, S. Y., and Mackey, D. 2007. Layered basal defenses underlie non-host resistance of Arabidopsis to Pseudomonas syringae pv. phaseolicola. Plant J. 51:604-616.

Hann, D. R., and Rathjen, J. P. 2007. Early events in the pathogenicity of Pseudomonas syringae on Nicotiana benthamiana. Plant J. 49:607618

Hann, D. R., and Rathjen, J. P. 2010. The long and winding road: Virulence effector proteins of plant pathogenic bacteria. Cell Mol. Life Sci. 67:3425-3434.

Hann, D. R., Gimenez-Ibanez, S., and Rathjen, J. P. 2010. Bacterial virulence effectors and their activities. Curr. Opin. Plant Biol. 13:388-393.

He, P., Shan, L., Lin, N. C., Martin, G. B., Kemmerling, B., Nurnberger, T., and Sheen, J. 2006. Specific bacterial suppressors of MAMP signaling upstream of MAPKKK in Arabidopsis innate immunity. Cell 125:563-575.

He, P., Shan, L., and Sheen, J. 2007. The use of protoplasts to study innate immune responses. Methods Mol. Biol. 354:1-9.

Heese, A., Hann, D. R., Gimenez-Ibanez, S., Jones, A. M., He, K., Li, J. Schroeder, J. I., Peck, S. C., and Rathjen, J. P. 2007. The receptor-like kinase SERK3/BAK1 is a central regulator of innate immunity in plants. Proc. Natl. Acad. Sci. U.S.A. 104:12217-12222.

Jackson, R. W., Athanassopoulos, E., Tsiamis, G., Mansfield, J. W., Sesma, A., Arnold, D. L., Gibbon, M. J., Murillo, J., Taylor, J. D., and Vivian, A. 1999. Identification of a pathogenicity island, which contains genes for virulence and avirulence, on a large native plasmid in the bean pathogen Pseudomonas syringae pathovar phaseolicola. Proc. Natl. Acad. Sci. U.S.A. 96:10875-10880.

Jamir, Y., Guo, M., Oh, H. S., Petnicki-Ocwieja, T., Chen, S., Tang, X., Dickman, M. B., Collmer, A., and Alfano, J. R. 2004. Identification of Pseudomonas syringae type III effectors that can suppress programmed cell death in plants and yeast. Plant J. 37:554-565.
Jones, J. D., and Dangl, J. L. 2006. The plant immune system. Nature 444:323-329.

Kim, J. G., Li, X., Roden, J. A., Taylor, K. W., Aakre, C. D., Su, B. Lalonde, S., Kirik, A., Chen, Y., Baranage, G., McLane, H., Martin, G. B., and Mudgett, M. B. 2009. Xanthomonas T3S effector XopN suppresses PAMP-triggered immunity and interacts with a tomato atypical receptor-like kinase and TFT1. Plant Cell 21:1305-1323.

Kim, M. G., da Cunha, L., McFall, A. J., Belkhadir, Y., DebRoy, S., Dangl, J. L., and Mackey, D. 2005. Two Pseudomonas syringae type III effectors inhibit RIN4-regulated basal defense in Arabidopsis. Cell 121:749759.

Lewis, J. D., Guttman, D. S., and Desveaux, D. 2009. The targeting of plant cellular systems by injected type III effector proteins. Semin. Cell Dev. Biol. 20:1055-1063.

Li, J., Wen, J., Lease, K. A., Doke, J. T., Tax, F. E., and Walker, J. C. 2002 BAK1, an Arabidopsis LRR receptor-like protein kinase, interacts with BRI1 and modulates brassinosteroid signaling. Cell 110:213-222.

Li, X., Lin, H., Zhang, W., Zou, Y., Zhang, J., Tang, X., and Zhou, J. M. 2005. Flagellin induces innate immunity in nonhost interactions that is suppressed by Pseudomonas syringae effectors. Proc. Natl. Acad. Sci. U.S.A. 102:12990-12995.

Lindeberg, M., Cartinhour, S., Myers, C. R., Schechter, L. M., Schneider, D. J., and Collmer, A. 2006. Closing the circle on the discovery of genes encoding Hrp regulon members and type III secretion system effectors in the genomes of three model Pseudomonas syringae strains. Mol. Plant-Microbe Interact. 19:1151-1158.

Lindeberg, M., Cunnac, S., and Collmer, A. 2009. The evolution of Pseudomonas syringae host specificity and type III effector repertoires. Mol. Plant Pathol. 10:767-775.

Liu, J., Elmore, J. M., Fuglsang, A. T., Palmgren, M. G., Staskawicz, B. J., and Coaker, G. 2009. RIN4 functions with plasma membrane $\mathrm{H}^{+}$ ATPases to regulate stomatal apertures during pathogen attack. PLoS Biol 7:e1000139. Published online.

Lu, D., Wu, S., Gao, X., Zhang, Y., Shan, L., and He, P. 2010. A receptorlike cytoplasmic kinase, BIK1, associates with a flagellin receptor complex to initiate plant innate immunity. Proc. Natl. Acad. Sci. U.S.A. 107:496-501.

Mackey, D., Holt, B. F., 3rd, Wiig, A., and Dangl, J. L. 2002. RIN4 interacts with Pseudomonas syringae type III effector molecules and is required for RPM1-mediated resistance in Arabidopsis. Cell 108:743754.

Mackey, D., Belkhadir, Y., Alonso, J. M., Ecker, J. R., and Dangl, J. L. 2003. Arabidopsis RIN4 is a target of the type III virulence effector AvrRpt 2 and modulates RPS2-mediated resistance. Cell 112:379-389.

McGhie, E. J., Brawn, L. C., Hume, P. J., Humphreys, D., and Koronakis, V. 2009. Salmonella takes control: effector-driven manipulation of the host. Curr. Opin. Microbiol. 12:117-124.

McNellis, T. W., Mudgett, M. B., Li, K., Aoyama, T., Horvath, D., Chua, N. H., Staskawicz, B. J. 1998. Glucocorticoid-inducible expression of a bacterial avirulence gene in transgenic Arabidopsis induces hypersensitive cell death. Plant J. 14:247-257.

Meng, X., Bonasera, J. M., Kim, J. F., Nissinen, R. M., and Beer, S. V. 2006. Apple proteins that interact with DspA/E, a pathogenicity effector of Erwinia amylovora, the fire blight pathogen. Mol. Plant-Microbe Interact. 19:53-61.

Nam, K. H., and Li, J. 2002. BRI1/BAK1, a receptor kinase pair mediating brassinosteroid signaling. Cell 110:203-212.

Nimchuk, Z., Marois, E., Kjemtrup, S., Leister, R. T., Katagiri, F., and Dangl, J. L. 2000. Eukaryotic fatty acylation drives plasma membrane targeting and enhances function of several type III effector proteins from Pseudomonas syringae. Cell 101:353-363.

Nomura, K., Debroy, S., Lee, Y. H., Pumplin, N., Jones, J., and He, S. Y. 2006. A bacterial virulence protein suppresses host innate immunity to cause plant disease. Science 313:220-223.

Oh, H. S., and Collmer, A. 2005. Basal resistance against bacteria in Nicotiana benthamiana leaves is accompanied by reduced vascular staining and suppressed by multiple Pseudomonas syringae type III secretion system effector proteins. Plant J. 44:348-359.

Raffatellu, M., Wilson, R. P., Chessa, D., Andrews-Polymenis, H., Tran, Q T., Lawhon, S., Khare, S., Adams, L. G., and Baumler, A. J. 2005. SipA, SopA, SopB, SopD, and SopE2 contribute to Salmonella enterica serotype typhimurium invasion of epithelial cells. Infect. Immun 73:146-154.

Robert-Seilaniantz, A., Shan, L., Zhou, J.-M., and Tang, X. 2006. The Pseudomonas syringae pv. tomato DC3000 type III effector HopF2 has a putative myristoylation site required for its avirulence and virulence functions. Mol. Plant-Microbe Interact. 19:130-138.

Rodriguez, M. C., Petersen, M., and Mundy, J. 2010. Mitogen-activated protein kinase signaling in plants. Annu. Rev. Plant Biol. 61:621-649.

Schulze, B., Mentzel, T., Jehle, A. K., Mueller, K., Beeler, S., Boller, T., 
Felix, G., and Chinchilla, D. 2010. Rapid heteromerization and phosphorylation of ligand-activated plant transmembrane receptors and their associated kinase BAK1. J. Biol. Chem. 285:9444-9451.

Shan, L., Thara, V. K., Martin, G. B., Zhou, J. M., and Tang, X. 2000. The pseudomonas AvrPto protein is differentially recognized by tomato and tobacco and is localized to the plant plasma membrane. Plant Cell 12:2323-2338.

Shan, L., Oh, H. S., Chen, J., Guo, M., Zhou, J., Alfano, J. R., Collmer, A., Jia, X., and Tang, X. 2004. The HopPtoF locus of Pseudomonas syringae pv. tomato DC3000 encodes a type III chaperone and a cognate effector. Mol. Plant-Microbe Interact. 17:447-455.

Shan, L., He, P., Li, J., Heese, A., Peck, S..C., Nurnberger, T., Martin, G..B., and Sheen, J. 2008. Bacterial effectors target the common signaling partner BAK1 to disrupt multiple MAMP receptor-signaling complexes and impede plant immunity. Cell Host Microbe 4:17-27.

Singer, A. U., Desveaux, D., Betts, L., Chang, J. H., Nimchuk, Z., Grant, S. R., Dangl, J. L., and Sondek, J. 2004. Crystal structures of the type III effector protein AvrPphF and its chaperone reveal residues required for plant pathogenesis. Structure 12:1669-1681.

Speth, E. B., Lee, Y. N., and He, S. Y. 2007. Pathogen virulence factors as molecular probes of basic plant cellular functions. Curr. Opin. Plant Biol. 10:580-586.

Suarez-Rodriguez, M. C., Adams-Phillips, L., Liu, Y., Wang, H., Su, S. H., Jester, P. J., Zhang, S., Bent, A. F., and Krysan, P. J. 2007. MEKK1 is required for flg22-induced MPK4 activation in Arabidopsis plants. Plant Physiol. 143:661-669.

Tsiamis, G., Mansfield, J. W., Hockenhull, R., Jackson, R. W., Sesma, A., Athanassopoulos, E., Bennett, M. A., Stevens, C., Vivian, A., Taylor, J. D., and Murillo, J. 2000. Cultivar-specific avirulence and virulence functions assigned to avrPphF in Pseudomonas syringae pv. phaseolicola, the cause of bean halo-blight disease. EMBO (Eur. Mol. Biol. Organ.) J. 19:3204-3214.

Veronese, P., Nakagami, H., Bluhm, B., Abuqamar, S., Chen, X., Salmeron, J., Dietrich, R. A., Hirt, H., and Mengiste, T. 2006. The membraneanchored BOTRYTIS-INDUCED KINASE1 plays distinct roles in Arabidopsis resistance to necrotrophic and biotrophic pathogens. Plant Cell 18:257-273.

Wang, Y., Li, J., Hou, S., Wang, X., Li, Y., Ren, D., Chen, S., Tang, X., and Zhou, J. M. 2010. A Pseudomonas syringae ADP-ribosyltransferase inhibits Arabidopsis mitogen-activated protein kinase kinases. Plant Cell 22:2033-2044.

Wilton, M., Subramaniam, R., Elmore, J., Felsensteiner, C., Coaker, G. and Desveaux, D. 2010. The type III effector HopF2Pto targets Arabidopsis RIN4 protein to promote Pseudomonas syringae virulence. Proc. Natl. Acad. Sci. U.S.A. 107:2349-2354.

Xiang, T., Zong, N., Zou, Y., Wu, Y., Zhang, J., Xing, W., Li, Y., Tang, X., Zhu, L., Chai, J., and Zhou, J. M. 2008. Pseudomonas syringae effector AvrPto blocks innate immunity by targeting receptor kinases. Curr. Biol. 18:74-80.

Zhang, J., Li, W., Xiang, T., Liu, Z., Laluk, K., Ding, X., Zou, Y., Gao, M., Zhang, X., Chen, S., Mengiste, T., Zhang, Y., and Zhou, J. M. 2010. Receptor-like cytoplasmic kinases integrate signaling from multiple plant immune receptors and are targeted by a Pseudomonas syringae effector. Cell Host Microbe 7:290-301.

Zipfel, C., Kunze, G., Chinchilla, D., Caniard, A., Jones, J. D., Boller, T., and Felix, G. 2006. Perception of the bacterial PAMP EF-Tu by the receptor EFR restricts Agrobacterium-mediated transformation. Cell 125:749-760. 